\title{
Blockers for the stability number and the chromatic number
}
C. Bazgan *
C. Bentz ${ }^{\dagger}$
C. Picouleau ${ }^{\ddagger}$
B. Ries $\S$

\begin{abstract}
Given an undirected graph $G=(V, E)$ and two positive integers $k$ and $d$, we are interested in finding a set of edges (resp. non-edges) of size at most $k$ to delete (resp. to add) in such a way that the chromatic number (resp. stability number) in the resulting graph will decrease by at least $d$ compared to the original graph. We investigate these two problems in various classes of graphs (split graphs, threshold graphs, bipartite graphs and their complements) and determine their computational complexity. In some of the polynomial-time solvable cases, we also give a structural description of a solution.
\end{abstract}

Keywords: Blocker, chromatic number, stability number, bipartite graph, split graph, threshold graph.

July 12, 2013

*PSL, Université Paris-Dauphine, LAMSADE, CNRS UMR 7243 and Institut Universitaire de France, Paris (France). Email: bazgan@lamsade.dauphine.fr

${ }^{\dagger}$ CEDRIC - CNAM, Paris (France). Email: cedric.bentz@cnam.fr

${ }^{\ddagger}$ CEDRIC - CNAM, Paris (France). Email: christophe.picouleau@cnam.fr

${ }^{\S}$ PSL, Université Paris-Dauphine, LAMSADE, CNRS UMR 7243, Paris (France). Email: ries@lamsade.dauphine.fr 


\section{Introduction}

A general framework of the problems that we consider in this paper can be described as follows. Consider, in an undirected graph $G=(V, E)$, a family of combinatorial structures $U$, each one having a value $v(U)$. Such a $U$ will be called optimal if it optimizes $v(U)$. Our goal is to determine which edges (or non-edges) of $G$ are such that if we delete them (resp. add them), the value of an optimal combinatorial structure in the new graph has decreased by a given amount compared to the original graph $G$. These edges (resp. non-edges) can be considered as the most important elements in the graph $G=(V, E)$. Such questions may occur in problems related to safety and reliability. Notice that instead of deleting edges, we may delete vertices (also called nodes).

The problem that consists in determining a set of edges/nodes of minimum cardinality whose removal decreases the value of an optimal structure in the new graph by a given amount $d$ is referred to as the min edge/node d-blocker problem. A complementary problem consisting in finding a set of edges/nodes of size $k$ whose removal from the graph causes the largest value decrease is referred to in the literature as the $k$ most vital edges/nodes problem. In [17] we can find some applications of these types of problems.

The min edge/node $d$-blocker and $k$ most vital edges/nodes versions have been studied for several problems, including shortest path, minimum spanning tree, maximum flow, maximum matching, independent set, vertex cover, $p$-median and $p$-center, domination set (in this context the minimum cardinality of a 1-blocker is also called the bondage number [11]). The $k$ most vital edges problem with respect to shortest path was proved to be $N P$-hard [2] and the min edge/node $d$-blocker with respect to shortest path (resp. $k$ most vital edges/nodes with respect to shortest path) were proved to be not 1.36-approximable (resp. not 2-approximable) if $P \neq N P$ [14]. For minimum spanning tree, the $k$ most vital edges problem is $N P$-hard [12] and $O(\log k)$-approximable [12]. Moreover, min node $d$-blocker and $k$ most vital nodes with respect to minimum spanning tree were proved to be not approximable under several assumptions [6]. In [20] it is proved that $k$ most vital edges with respect to maximum flow is $N P$-hard. For maximum matching, $k$ most vital nodes was shown polynomialtime solvable for unweighted bipartite graphs and $N P$-hard for bipartite graphs when edge weights are bounded by a constant [21]. Moreover, min edge $d$-blocker with respect to maximum matching is $N P$-hard even for unweighted bipartite graphs [22], but polynomial for grids and trees [16]. The $k$ most vital nodes and min node $d$-blocker versions with respect to independent set for bipartite graphs remain polynomial on unweighted graphs [4, 10] and become NP-hard for weighted graphs [4]. For bounded treewidth graphs and cographs these versions remain polynomial [4]. Concerning the approximation on bipartite weighted graphs, $k$ most vital nodes with respect to independent set and vertex cover have no PTAS [3]. Min edge/node $d$-blocker and $k$ most vital edges/nodes with respect to $p$-median and $p$-center were proved to be not constant approximable, if $P \neq N P$ [5].

In this paper, all graphs are undirected and simple. Let $G=(V, E)$ be a graph. For a vertex $u \in V$, we let $N_{G}(u)$ denote the set of its neighbours in $G$ and we write $d_{G}(u)=\left|N_{G}(u)\right|$. If it is clear from the context what $G$ is, we will omit the subscript and simply write $N(u), d(u)$. The minimum and the maximum degree of $G$ are denoted by $\delta(G)$ and $\Delta(G)$, respectively. For any set $V^{\prime} \subseteq V$, we denote by $G\left[V^{\prime}\right]$ the subgraph induced by $V^{\prime}$. The length of a shortest cycle in $G$ is called the girth of $G$ and is denoted by $g(G)$. As usual, $C_{n}$ and $P_{n}$ denote the induced cycle on $n$ vertices and the induced path on $n$ vertices, respectively. $K_{n}$ denotes the complete graph of order $n$. We denote by $G-E^{\prime}$, for some subset $E^{\prime} \subseteq E$, the graph obtained from $G$ by deleting the edges of $E^{\prime}$. The complement of a graph $G=(V, E)$ is denoted by $\bar{G}=(V, \bar{E})$. Let $\bar{E}^{\prime}$ be a set of non-edges of a graph $G=(V, E)$ (i.e., $\bar{E}^{\prime}$ is a set of edges in $\left.\bar{G}\right)$. Then we denote by $G+\bar{E}^{\prime}$ the graph obtained from $G$ by transforming the non-edges of $\bar{E}^{\prime}$ into edges. If $G_{1}$ and $G_{2}$ are two graphs we denote by $G_{1} \cup G_{2}$ their disjoint union. In the remainder of the paper, whenever a graph $G$ is given, $V(G)$ and $E(G)$ respectively denote its 
vertex and edge sets.

A matching $M$ of $G$ is a set of pairwise non-adjacent edges. The largest cardinality of a matching in $G$ is called the matching number of $G$ and denoted by $\mu(G)$. If for a vertex $u$, there exists an edge $u v \in M, u$ is said to be saturated by $M$. A matching in $G$ is called perfect if all vertices are saturated. A path $P=\left(e_{1}, e_{2}, \ldots, e_{p}\right)$ (resp. even cycle $C$ ) is called alternating with respect to a matching $M$, if $e_{i} \in M, e_{i+1} \notin M$ for $i=1, \ldots, p-1$ or $e_{i} \notin M, e_{i+1} \in M$ for $i=1, \ldots, p-1$.

A $k$-coloring is a mapping $c: V \rightarrow\{1, \ldots, k\}$ such that $c(u) \neq c(v)$ for every edge $u v$ in $E$. If such a mapping exists, $G$ is said to be $k$-colorable. The chromatic number $\chi(G)$ of $G$ is the smallest integer $k$ such that $G$ is $k$-colorable. A set $E^{\prime} \subseteq E$ such that $\chi\left(G-E^{\prime}\right) \leq \chi(G)-d$, where $d$ is an integer, is called a chromatic d-blocker. A minimum chromatic $d$-blocker is a chromatic $d$-blocker of minimum size. The chromatic d-blocker number $c b_{d}(G)$ of a graph $G$ is the size of a minimum chromatic $d$-blocker.

From the definition of a minimum chromatic $d$-blocker the following remark trivially holds.

Remark 1.1 Let $G=(V, E)$ be a graph and $B \subseteq E$ a minimum chromatic d-blocker: then we have $\chi(G-B)=\chi(G)-d$.

A stable set $S$ of $G$ is a set of pairwise non-adjacent vertices. The largest cardinality of a stable set in $G$ is called the stability number of $G$ and denoted by $\alpha(G)$. A set $\bar{E}^{\prime}$ of non-edges of $G$ such that $\alpha\left(G+\bar{E}^{\prime}\right) \leq \alpha(G)-d$, where $d$ is an integer, is called a stability d-blocker. A minimum stability $d$-blocker is a stability $d$-blocker of minimum size. The stability $d$-blocker number $s b_{d}(G)$ of a graph $G$ is the size of a minimum stability $d$-blocker.

We are interested in the following two decision problems and their optimization versions.

\section{Chromatic $d$-Blocker}

Input: A graph $G=(V, E)$, a positive integer $d$, and an integer $0 \leq k \leq|E|$.

Question: Does there exist a set $B \subseteq E$ such that $|B| \leq k$ and $\chi(G-B) \leq \chi(G)-d$ ?

\section{StABILITY $d$-BLOCKER}

Input: A graph $G=(V, E)$, a positive integer $d$, and an integer $0 \leq k \leq|\bar{E}|$.

Question: Does there exist a set of non-edges $\bar{B} \subseteq \bar{E}$ such that $|\bar{B}| \leq \bar{k}$ and $\alpha(G+\bar{B}) \leq \alpha(G)-d$ ?

We will use Chromatic $d$-Blocker and Stability $d$-Blocker indifferently for the decision and the optimization problems. Notice that both problems may not be in NP: indeed, determining the chromatic number and the stability number of a graph are $N P$-hard problems, and hence there is no easy way to prove their membership of the class $N P$.

We may assume for Chromatic $d$-Blocker that $\chi(G) \geq 3$. Indeed, if $\chi(G) \leq 2$ then $G$ is bipartite and hence we need to delete all edges of $G$ in order to decrease the chromatic number. For similar reasons, we may assume for STABILITY $d$-BLOCKER that $\alpha(G) \geq 3$.

In what follows, we will analyze the complexity of these two problems in various well-known graph classes. Notice that whenever $G$ belongs to some graph class $\mathcal{G}$, we do not impose that the resulting graph $G-B$, respectively $G+\bar{B}$, belongs to $\mathcal{G}$ as well. Indeed, for the graph classes that we consider here, these problems become easy to solve if we do impose this constraint as can easily be verified.

The rest of the paper is organized as follows. In Section 2 some complexity results are established on complete graphs and their complements, and on general graphs. In Section 3 we consider split graphs and a particular subclass, the threshold graphs. After considering the complements of bipartite graphs in Section 4, we study the bipartite graphs in Section 5. A table with the results obtained in the paper as well as some open problems are provided in Section 6. 


\section{Preliminary results}

We recall that the Turán graph $T_{n, r}$ is the complete $r$-partite graph on $n$ vertices whose partition sets differ in size by at most 1 (i.e., all partition sets have size $\left\lfloor\frac{n}{r}\right\rfloor$ or $\left\lceil\frac{n}{r}\right\rceil$ ). Turán proved that $T_{n, r}$ has the maximum number of edges among all graphs of order $n$ with no complete graph $K_{r+1}$ as a subgraph [18]. In other words, since $T_{n, r}$ is $r$-colorable, every graph $H$ with $|V(H)|=\left|V\left(T_{n, r}\right)\right|=n$ and $|E(H)|>\left|E\left(T_{n, r}\right)\right|=\left\lfloor\frac{(r-1) n^{2}}{2 r}\right\rfloor$ has $\chi(H)>\chi\left(T_{n, r}\right)=r$ (see [19]). Moreover it is well known that $T_{n, r}$ is a perfect graph. Thus we immediately obtain the following.

Corollary 2.1 Chromatic $d$-Blocker is polynomial-time solvable for complete graphs $K_{n}$. A minimum chromatic d-blocker consists of $E\left(\bar{T}_{n, n-d}\right)$ and $c b_{d}\left(K_{n}\right)=\left(\begin{array}{l}n \\ 2\end{array}\right)-\left\lfloor\frac{(n-d-1) n^{2}}{2(n-d)}\right\rfloor$.

Corollary 2.2 StABILITy $d$-BLOCKER is polynomial-time solvable for the complements of complete graphs. A minimum stability d-blocker in the complement of a complete graph $K_{n}$ consists of $E\left(\bar{T}_{n, n-d}\right)$, and $s b_{d}\left(\bar{K}_{n}\right)=\left(\begin{array}{l}n \\ 2\end{array}\right)-\left\lfloor\frac{(n-d-1) n^{2}}{2(n-d)}\right\rfloor$.

Now let us establish our first two results concerning the complexity of our two problems in general graphs when $d$ is fixed.

Theorem 2.3 For every fixed $d \geq 1$, Chromatic $d$-Blocker is NP-hard.

Proof: We use a reduction from the $N P$-hard problem Bipartite SUBGRAPH which is defined as follows (see [13]): given a graph $G=(V, E)$ with $\Delta(G) \leq 3$ and an integer $t \leq|E|$, decide whether there exists a subset $E^{\prime} \subseteq E$ with $\left|E^{\prime}\right| \leq t$ and such that $G-E^{\prime}$ is bipartite. It follows from Brook's theorem (see [8]) that we may assume that $\chi(G) \leq 3$. Furthermore, since deciding if $G$ has $\chi(G)=2$ can be done in polynomial time and in this case BIPARTITE SUBGRAPH is trivial, we may actually assume that $\chi(G)=3$.

Consider an instance $I$ of Bipartite subgraph, i.e., a graph $G$ with $\Delta(G) \leq 3$ and an integer $t \leq|E|$. Let $\tilde{G}=G \cup K_{d+2}$ and $k=t+c b_{d}\left(K_{d+2}\right)=t+\left(\begin{array}{c}d+2 \\ 2\end{array}\right)-\left\lfloor\frac{(d+2)^{2}}{4}\right\rfloor$. This gives us an instance $I^{\prime}$ of Chromatic $d$-BLOCKer and the reduction can be done in polynomial time. Clearly $\chi(\tilde{G})=d+2$ (recall that $\chi(G)=3$ ).

Now if $I$ is a yes-instance, let $E^{\prime} \subseteq E,\left|E^{\prime}\right| \leq t$ such that $G-E^{\prime}$ is a bipartite graph. It follows from Corollary 2.1 that if we delete from $K_{d+2}$ the edges of $\bar{T}_{d+2,2}$ we obtain a graph $H$ with $\chi(H) \leq d+2-d=2$. Thus by taking $B=E^{\prime} \cup E\left(\bar{T}_{d+2,2}\right)$, it follows that $|B| \leq k$ and $\chi(\tilde{G}-B) \leq \chi(\tilde{G})-d$. Hence $I^{\prime}$ is a yes-instance.

Conversely, suppose that $I^{\prime}$ is a yes-instance and let $B \subseteq E(\tilde{G})$ with $|B| \leq k$ such that $\chi(\tilde{G}-B) \leq$ $\chi(\tilde{G})-d=2$. From Corollary 2.1 it follows that we need to delete $c b_{d}\left(K_{d+2}\right)$ edges from $K_{d+2}$. Since $\chi(\tilde{G})-d=2$, it follows that $G-(B \cap E)$ is bipartite and $|B \cap E| \leq t$. Hence by taking $E^{\prime}=B \cap E$, we deduce that $I$ is a yes-instance.

Theorem 2.4 For every fixed $d \geq 1$, StABILITy $d$-BLOCKER is NP-hard.

Proof: We use a reduction from the maximum stable set problem which is $N P$-hard. In this problem, we are given a graph $G=(V, E)$, and we want to determine the size $\alpha(G)$ of a maximum stable set in $G$. Given an integer $d \geq 1$, we construct $|V|$ instances of Stability $d$-BLOCKER as follows: $H_{i}$, the graph of the $i$ th of these instances, consists of a copy $G_{i}$ of the graph $G$ and of a stable set $S_{i}$ with 
$d+i$ vertices; moreover, we add an edge between every vertex of $S_{i}$ and every vertex of $G_{i}$. Notice that, because of all the edges we added, for any set of non-edges $B$, a maximum stable set in $H_{i}+B$ is either entirely contained in $S_{i}+B \cap E\left(\bar{S}_{i}\right)$ or entirely contained in $G_{i}+B \cap E\left(\bar{G}_{i}\right)$. Finally, for each $i$, we ask whether there exists a stability $d$-blocker of size at most $\left|E\left(\bar{T}_{d+i, i}\right)\right|$ in $H_{i}$. Now, assume that there exists a polynomial-time algorithm $\mathcal{A}$ for solving STABILITy $d$-BLOCKER. We run Algorithm $\mathcal{A}$ on all the instances $H_{i}$, for $i$ from $|V|$ to 1 (in this order). We claim that the first instance $H_{j}$ for which the answer of $\mathcal{A}$ is no is such that $j=\alpha(G)-1$, and so Algorithm $\mathcal{A}$ would enable us to compute $\alpha(G)$ in polynomial time, which is impossible unless $P=N P$.

Indeed, if $i \geq \alpha(G)$, then $\alpha\left(H_{i}\right)=i+d \geq \alpha(G)+d$, and thus $\alpha\left(H_{i}\right)-d \geq \alpha(G)$. Hence, when computing a stability $d$-blocker for $H_{i}$, there is no need to decrease the stability number of $G_{i}$, and only the one of $S_{i}$ must decrease by $d$ units. So, from Corollary 2.2, Algorithm $\mathcal{A}$ must answer yes. Now, if $i=\alpha(G)-1$, then $\alpha\left(H_{i}\right)=\alpha(G)+d-1 \geq \alpha(G)$, and thus $\alpha\left(H_{i}\right)-d=\alpha(G)-1$. Hence, when computing a stability $d$-blocker for $H_{i}$, we must both decrease the stability number of $S_{i}$ by $d$ units (which, from Corollary 2.2, cannot be done by adding less than $\left|E\left(\bar{T}_{d+i, i}\right)\right|$ edges) and the stability number of $G_{i}$ by one unit (which requires to add at least one edge). Therefore, any such stability blocker for $H_{i}$ will use at least $\left|E\left(\bar{T}_{d+i, i}\right)\right|+1$ edges, and Algorithm $\mathcal{A}$ must answer no.

\section{Split graphs}

A split graph $G=(V, E)$ is a graph whose vertex set $V$ can be partitioned into two subsets: one inducing a stable set $S$ and the other one inducing a clique $K$. We will give the following two results for the case when $d$ is fixed.

Theorem 3.1 For every fixed $d \geq 1$, Chromatic $d$-BLOCKER is polynomial-time solvable in split graphs.

Proof: Let $G=(V, E)$ be a split graph with stable set $S$ and clique $K$ and let $d \geq 1$ be a fixed integer. We assume without loss of generality that $\chi(G)=|V(K)|$ (i.e., $V(K)$ is maximal). Notice that we can suppose that $|V(K)| \geq d+2$, the case where $|V(K)|=d+1$ being trivial ( $E$ is the unique chromatic $d$-blocker), and for $|V(K)| \leq d$ there exists no chromatic $d$-blocker. We will distinguish two cases:

(i) $|V(K)| \geq 2(d+1)$

This implies that there exists a matching $M$ of size $d+1$ in $K$. By deleting the edges of $M$ we obtain a graph $G^{\prime}$ which is $(\chi(G)-d)$-colorable. Indeed, for every edge $u_{i} v_{i} \in M$, $i=1, \ldots, d+1$, we color $u_{i}$ and $v_{i}$ with a same color $c_{i}$; then we color the remaining vertices of $K$ with $|V(K)|-2 d-2$ new colors and finally we use another new color for all the vertices in $S$. This clearly gives us a $(|V(K)|-d)$-coloring of $G^{\prime}$. Thus $M$ is a chromatic $d$-blocker of $G$. Furthermore, for every subset $B \subseteq E$ with $|B|<d+1$, we check whether $B$ is a chromatic $d$-blocker of $G$. Since $d$ is fixed, we have a polynomial number of sets $B$ to consider. If no chromatic $d$-blocker $B$ of $G$ exists with $|B|<d+1$, then $M$ is an optimal solution. Otherwise, we take a smallest chromatic $d$-blocker $B$ of $G$ with $|B|<d+1$.

(ii) $|V(K)|<2(d+1)$

Since $|V(K)| \geq d+2$ the edge set $E(K)$ is a chromatic $d$-blocker of $G$ of size at most $d(2 d+1)$. Similar to the previous case, we check for every subset $B \subseteq E$ with $|B|<d(2 d+1)$ whether $B$ is a chromatic $d$-blocker of $G$. If no such set $B$ exists, then $E(K)$ is an optimal solution. Otherwise we take a smallest chromatic $d$-blocker $B$ of $G$ with $|B|<d(2 d+1)$. 
Theorem 3.2 For every fixed $d \geq 1$, STABILITy $d$-BLOCKER is polynomial-time solvable in split graphs.

Proof: Let $G$ be a split graph with clique $K$ and stable set $S$. We assume without loss of generality that $|V(S)|=\alpha(G)$ (i.e., $V(S)$ is maximal). If $\alpha(G) \leq d$, then clearly there exists no solution. If $\alpha(G)=d+1$, then the only optimal solution consists in transforming $G$ into a clique. So we may assume now that $\alpha(G) \geq d+2$. In that case we choose $d+2$ vertices in $V(S)$ and add all edges between them, i.e., we transform $\frac{(d+2)(d+1)}{2}$ non-edges into edges. This clearly gives us a graph with stability number at most $\alpha(G)-d$, and thus the non-edges form a stability $d$-blocker. Hence an optimal solution consists of at most $\frac{(d+2)(d+1)}{2}$ non-edges and since $d$ is fixed, we can find such a solution in polynomial time.

This settles the case where $d$ is fixed in split graphs. This leaves as open the complexity of both problems in split graphs, when $d$ is given as part of the input.

Now we will be interested in a subclass of split graphs, namely threshold graphs. A threshold graph $G=(V, E)$ is a split graph with the following property: the vertices of $S$ can be ordered $v_{1}, \ldots, v_{p}$ such that $N_{G}\left(v_{1}\right) \subseteq N_{G}\left(v_{2}\right) \subseteq \ldots \subseteq N_{G}\left(v_{p}\right)$. We denote by $u_{1}, \ldots, u_{q}$ the vertices of $K$, and we suppose that $d_{G}\left(u_{1}\right) \leq d_{G}\left(u_{2}\right) \leq \ldots \leq d_{G}\left(u_{q}\right)$.

We will show that Chromatic $d$-BLOCKER is polynomial-time solvable for threshold graphs. We begin with the following lemma.

Lemma 3.3 Let $G=(V, E)$ be a threshold graph and let $B^{\prime} \subseteq E$ be a chromatic d-blocker such that $\chi\left(K-\left(B^{\prime} \cap E(K)\right)\right)=q-d$. Let $C_{1}^{\prime}, \ldots, C_{q-d}^{\prime}$ be the color classes of an optimal coloring of $K-\left(B^{\prime} \cap E(K)\right)$ with $\left|C_{1}^{\prime}\right| \leq\left|C_{2}^{\prime}\right| \leq \ldots \leq\left|C_{q-d}^{\prime}\right|$. Then there exists a chromatic d-blocker $B \subseteq E$ such that

(1) $|B| \leq\left|B^{\prime}\right|$;

(2) the color classes $C_{1}, \ldots, C_{q-d}$ of an optimal coloring of $K-(B \cap E(K))$ satisfy $\left|C_{i}\right|=\left|C_{i}^{\prime}\right|$, for $i=1, \ldots, q-d$;

(3) $C_{1}=\left\{u_{1}, \ldots, u_{\left|C_{1}^{\prime}\right|}\right\}$;

(4) $B$ contains all edges between $V(S)$ and $C_{1}$.

Proof: First notice that $B^{\prime}$ contains all edges between vertices of a same set $C_{i}^{\prime}$, for $i=1, \ldots, q-d$. In addition, since $G-B^{\prime}$ is $(q-d)$-colorable, for every vertex $v_{j} \in V(S)$, there exists one set $C_{i}^{\prime}$ such that $B^{\prime}$ contains all edges of $E$ between $C_{i}^{\prime}$ and $v_{j}$, for $i \in\{1, \ldots, q-d\}$ and $j=1, \ldots, p$. Now we construct the chromatic $d$-blocker $B$ as follows. We partition the vertices of $K$ into $q-d$ sets $K^{1}, \ldots, K^{q-d}$ such that $K^{1}=\left\{u_{1}, \ldots, u_{\left|C_{1}^{\prime}\right|}\right\}$ and $\left|K^{i}\right|=\left|C_{i}^{\prime}\right|$ for $i=2, \ldots, q-d$ (the vertices in $K^{i}$, $i \neq 1$, can be chosen arbitrarily). Now we put into $B$ all edges between vertices of a same set $K^{i}$, for $i=1, \ldots, q-d$ and all edges between $V(S)$ and $K^{1}$. Clearly $K-(B \cap E(K))$ is $(q-d)$-colorable (simply take the color classes $C_{i}=K^{i}$ for $i=1, \ldots, q-d$ ). Furthermore, since there are no more edges between $V(S)$ and $C_{1}$, all vertices of $S$ may be colored with the same color as the vertices in $C_{1}$. Thus $B$ is a chromatic $d$-blocker of $G$ satisfying (2), (3) and (4). It remains to show that $|B| \leq\left|B^{\prime}\right|$. Notice that, since, for each $i,\left|C_{i}\right|=\left|C_{i}^{\prime}\right|$, we have $\left|B^{\prime} \cap E(K)\right| \leq|B \cap E(K)|$. As already mentioned before, for every vertex $v_{j} \in V(S)$, there exists one set $C_{i}^{\prime}$ such that $B^{\prime}$ contains all edges of $E$ between $C_{i}^{\prime}$ and $v_{j}$, for $i \in\{1, \ldots, q-d\}$ and $j=1, \ldots, p$. Also notice that since $G$ is a threshold graph, if a vertex $v_{j}$ is adjacent to some vertex $u_{i}, i \in\{1, \ldots, q\}$, then $v_{j}$ is adjacent to all vertices $u_{\ell}$ for 
$\ell=i, i+1, \ldots, q$. Now consider some arbitrary vertex $v_{j} \in V(S)$. Suppose that $v_{j}$ is adjacent to some vertex $u_{i}$ in $G$ for $i \in\left\{2, \ldots,\left|C_{1}\right|\right\}$ and nonadjacent to $u_{i-1}$. Thus $B$ contains all edges $v_{j} u_{\ell}$ for $\ell=i, i+1, \ldots,\left|C_{1}\right|$. Let $C_{r}^{\prime}$ be the color class such that $B^{\prime}$ contains all edges of $E$ between $C_{r}^{\prime}$ and $v_{j}$. Since $G$ is a threshold graph, $v_{j}$ is adjacent to all vertices of $K$ except $u_{1}, \ldots, u_{i-1}$. Thus $B^{\prime}$ must contain at least $\left|C_{r}^{\prime}\right|-i+1$ edges of $E$ between $C_{r}^{\prime}$ and $v_{j}$. But since $\left|C_{r}^{\prime}\right| \geq\left|C_{1}\right|$ it follows that $\left|C_{r}^{\prime}\right|-i+1 \geq\left|C_{1}\right|-i+1$. Notice that when $v_{j}$ is adjacent to all vertices in $V(K), B^{\prime}$ contains $\left|C_{r}^{\prime}\right|$ (resp. $\left|C_{1}\right|$ ) edges of $E$ between $v_{j}$ and $C_{r}$ (resp. $C_{1}$ ). Hence, in both cases, $B^{\prime}$ contains at least as many edges incident to $v_{j}$ as $B$. Since this is true for all vertices of $S$, we deduce that $|B| \leq\left|B^{\prime}\right|$.

Remark 3.1 It follows from the proof of Lemma 3.3 that if $B$ is a minimum chromatic d-blocker for a threshold graph $G$ with $\chi(K-(B \cap E(K)))=q-d$, we may assume that $B$ consists of all edges between $V(S)$ and $C_{1}$, as well as of all edges between vertices of $K$ which belong to the same color class $C_{j}$, for $j=1, \ldots, q-d$. Clearly, $B$ does not contain any other edge. Furthermore, it follows from Corollary 2.1 that $|B|=\frac{\left|C_{1}\right|\left(\left|C_{1}\right|-1\right)}{2}+\left|E\left(\bar{T}_{q-\left|C_{1}\right|, q-d-1}\right)\right|+\left|E\left(C_{1}, S\right)\right|$, where $E\left(C_{1}, S\right)$ is the set of edges between vertices of $C_{1}$ and $S$.

Now we are ready to prove the following.

Theorem 3.4 Chromatic $d$-BLOCKer is polynomial-time solvable in threshold graphs.

Proof: Let $G=(V, E)$ be a threshold graph with clique $K$ and stable set $S$. Without loss of generality, we may assume that $V(K)$ is maximal. Let $B \subseteq E$ be a minimum chromatic $d$-blocker: recall that $\chi(G-B)=q-d$. Furthermore, we necessarily have one of the following cases:

(a) $\chi(K-(B \cap E(K)))=q-d-1$. Thus $B \subseteq E(K)$ and it immediately follows from Corollary 2.1 that $|B|=c b_{d+1}\left(K_{q}\right)=\frac{q(q-1)}{2}-\left|E\left(T_{q, q-d-1}\right)\right|$.

(b) $\chi(K-(B \cap E(K)))=q-d$. It follows from Lemma 3.3 that we may assume that $B$ contains all edges between the vertices of $C_{1}=\left\{u_{1}, \ldots, u_{i^{*}}\right\}$, for some $i^{*} \in\{1, \ldots, q\}$, all edges between $V(S)$ and $C_{1}$, as well as all edges between the vertices of $K$ belonging to a same color class $C_{j}$, for $j=2, \ldots, q-d$. Since we do not know the value of $i^{*}$, we will try all possible values of $i^{*}$, that is $1,2, \ldots,\left\lfloor\frac{q}{q-d}\right\rfloor$. Indeed, since $C_{1}$ is a smallest color class, its size cannot exceed $\left\lfloor\frac{q}{q-d}\right\rfloor$. Then, we keep the best solution obtained among these solutions, i.e., the one with the smallest total number of edges removed. Let us denote this number by $c b^{*}$.

Thus, we only need to compare $c b^{*}$ and $c b_{d+1}\left(K_{q}\right)$ in order to obtain a minimum chromatic $d$-blocker.

\section{Complements of bipartite graphs}

In this section, we will consider CHROMATIC $d$-BLOCKER in the case of complements of bipartite graphs. Notice that Chromatic $d$-BLocker in a graph $G=(V, E)$ is equivalent to asking whether in the complement graph $\bar{G}$ there exists a set of at most $k$ non-edges $\bar{B}$ such that transforming these non-edges into edges decreases the clique covering number $\theta(\bar{G})$ by at least $d$, i.e. $\theta(\bar{G}+\bar{B}) \leq \theta(\bar{G})-d$, where the clique covering number of a graph $G$ is the minimum number of cliques in $G$ such that every vertex belongs to at least one of these cliques. So we may define the following equivalent problem.

Clique Covering $d$-Blocker

Input: A graph $G=(V, E)$, a positive integer $d$, and an integer $0 \leq k \leq|\bar{E}|$. 
Question: Does there exist a set of non-edges $\bar{B} \subseteq \bar{E}$ such that $|\bar{B}| \leq k$ and $\theta(G+\bar{B}) \leq \theta(G)-d$ ?

Notice that finding a minimum clique covering in a bipartite graph $H$ with $n$ vertices consists in finding a maximum matching in $H$ since we have $\theta(H)=n-\mu(H)$. Indeed, a minimum clique covering of $H$ consists of the edges of a maximum matching (cliques of size 2) and the unsaturated vertices (cliques of size 1). We will use this fact in order to prove the following result which deals with the case $d=1$.

Theorem 4.1 Let $G=(V, E)$ be the complement of a bipartite graph with $|V|=n \geq 3$. Then

(a) $c b_{1}(G)=1$ if and only if

(a1) either $2 \mu(\bar{G}) \leq n-2$;

(a2) or $2 \mu(\bar{G})=n-1$ and $\bar{G}$ contains no isolated vertex;

(b) $c b_{1}(G)=2$ if and only if

(b1) either $2 \mu(\bar{G})=n-1$ and $\bar{G}$ contains one isolated vertex;

(b2) or $2 \mu(\bar{G})=n$ and $\bar{G}$ contains a $P_{6}$ whose first, third, and fifth edges belong to a perfect matching of $\bar{G}$;

(b3) or $2 \mu(\bar{G})=n$ and $\bar{G}$ contains a $C_{4}$ having two edges belonging to a perfect matching of $\bar{G}$;

(c) in all remaining cases:

(c1) $c b_{1}(G)=3$ if and only if at least one connected component of $\bar{G}$ contains at least four vertices;

(c2) $c b_{1}(G)=4$ otherwise.

Proof: Let $G=(V, E)$ be the complement of a bipartite graph with $|V|=n \geq 3$.

(a1) If $2 \mu(\bar{G}) \leq n-2$, then clearly for any maximum matching $M$ of $\bar{G}$ there exist two nonadjacent vertices $x, y$ in $\bar{G}$ which are not saturated by $M$. Thus adding the edge $x y$ increases the size of a maximum matching in $\bar{G}$ and hence decreases $\theta(\bar{G})$ by one.

(a2) If $2 \mu(\bar{G})=n-1$ then clearly for any maximum matching $M$ there exists exactly one vertex $x$ in $\bar{G}$ which is not saturated by $M$. If in addition $\bar{G}$ contains no isolated vertex, it follows that $x$ is adjacent to some vertex $y$ which is saturated by $M$. Let $z$ be such that $y z \in M$. Now by adding the edge $x z$, we clearly decrease $\theta(\bar{G})$ by one since a minimum clique covering consists now of $M \backslash\{y z\}$ and the triangle induced by $x, y, z$.

We need to add at least two edges to a bipartite graph to form a $K_{4}$ or two $K_{3}$. Moreover, if the graph has an isolated vertex $v$ then we need to add at least two edges to form a $K_{3}$ containing $v$. So in all remaining cases we have to add at least two edges in order to make the clique covering number of $\bar{G}$ decrease by at least one. We distinguish several cases:

(b1) $2 \mu(\bar{G})=n-1$ and $\bar{G}$ contains one isolated vertex. Let $M$ be a maximum matching. Let $x$ be the isolated vertex in $\bar{G}$. Consider an edge $y z \in M$. By adding the edges $x y, x z$ we obtain that $\theta(\bar{G}+\{x y, x z\}) \leq \theta(\bar{G})-1$.

So we may assume now that $\bar{G}$ contains no isolated vertex. Notice furthermore that from now on we may assume that $\bar{G}$ admits a perfect matching $M$.

(b2) $\bar{G}$ contains a path $P=\{x y, y z, z t, t u, u v\}$ such that $x y, z t, u v \in M$. Now, by adding the edges $x z, t v$ the vertices of $P$ are covered by two triangles and thus $\theta(\bar{G}+\{x z, t v\}) \leq \theta(\bar{G})-1$.

Thus we may assume now that $\bar{G}$ does not contain such a path $P$. 
(b3) $\bar{G}$ contains a cycle $C=\{x y, y z, z t, t x\}$ such that $x y, z t \in M$. Then by adding the edges $x z, y t$ we obtain a clique on four vertices and thus $\theta(\bar{G}+\{x z, y t\}) \leq \theta(\bar{G})-1$.

In [1] page 58, Corollary 5.1.8 states: Let a graph $G$ have a perfect matching $M$. Then any other perfect matching can be obtained from $M$ by a sequence of transfers along alternating cycles relative to $M$. From (b1), (b2) and (b3), $\bar{G}$ admits a perfect matching $M$, but no alternating cycle with respect to $M$. Hence, using the corollary, $\bar{G}$ admits a unique perfect matching.

Let us show now that in all remaining cases, we will need to add at least three edges in order to make the clique covering number of $\bar{G}$ decrease by at least one.

(c) First we claim the following: If $\bar{G}$ admits a unique perfect matching $M$ and does not contain a $P_{6}$ as described in (b2), then for every edge $x y \in M$, we have $d(x)=1$ or $d(y)=1$. Indeed, suppose that $d(x), d(y) \geq 2$. Let $x^{\prime}$ and $y^{\prime}$ be neighbors of $x$ and $y$, respectively. Since $M$ is perfect $x^{\prime}, y^{\prime}$ are both saturated. If $x^{\prime} y^{\prime} \in M$, then we obtain a $C_{4}$ as described in (b3), a contradiction (since $M$ would not be unique). Thus there exist $x^{\prime \prime}, y^{\prime \prime}$ such that $x^{\prime} x^{\prime \prime}, y^{\prime} y^{\prime \prime} \in M$. But now $\left\{x^{\prime \prime} x^{\prime}, x^{\prime} x, x y, y y^{\prime}, y^{\prime} y^{\prime \prime}\right\}$ is a $P_{6}$ as described in (b2), a contradiction. This proves the claim.

Thus $\bar{G}$ contains a stable set $S=\left\{x_{1}, \ldots, x_{\frac{n}{2}}\right\}$ such that $d\left(x_{i}\right)=1,1 \leq i \leq \frac{n}{2}$, and $\bar{G}$ has a unique minimum clique cover $\left\{x_{1}, y_{1}\right\}, \ldots,\left\{x_{\frac{n}{2}}, y_{\frac{n}{2}}\right\}$ that corresponds to its unique perfect matching $M=\left\{x_{1} y_{1}, \ldots, x_{\frac{n}{2}} y_{\frac{n}{2}}\right\}$. So we have $\theta(\bar{G})=\mu(\bar{G})$.

Suppose by contradiction that adding two edges gives us a graph $G^{\prime}$ such that $\theta\left(G^{\prime}\right) \leq \theta(\bar{G})-1=$ $\frac{n}{2}-1$. Clearly one of these two edges has its two extremities in $S$. Moreover, since $\bar{G}$ is bipartite $G^{\prime}$ has to contain a triangle, so exactly one of the two edges has its endpoints in $S$, say $x_{i}$ and $x_{j}$. Hence there is a minimum clique cover of $G^{\prime}$ which contains all the $\left\{x_{k}, y_{k}\right\}, k \neq i, j$. Since $\theta\left(G^{\prime}\right) \leq \frac{n}{2}-1$ the four vertices $x_{i}, y_{i}, x_{j}, y_{j}$ must induce a $K_{4}$ in $G^{\prime}$ but this requires to add at least three edges to $\bar{G}$, a contradiction.

Thus we conclude that we need to add at least three edges in order to make the clique covering number of $\bar{G}$ decrease by at least one.

(c1) Suppose that at least one connected component $C$ of $\bar{G}$ contains at least four vertices. Since $\bar{G}$ admits a unique perfect matching $M$, we may assume that $x, y, z, t$ belong to $C$ with $x y, z t, x t$ being edges in $C$ and $x y, z t \in M$. Since $M$ is unique, it follows that $y, z$ are nonadjacent. Thus by adding the edges $y z, x z, y t$, we obtain a clique on four vertices. Clearly $\theta(\bar{G}+\{y z, x z, y t\}) \leq$ $\theta(\bar{G})-1$.

(c2) The remaining case corresponds to $\bar{G}$ being isomorphic to $\frac{n}{2} K_{2}$. Let $x y, z t \in M$. By adding the four edges $x z, x t, y z, y t$, we clearly obtain that $\theta(\bar{G}+\{x z, x t, y z, y t\}) \leq \theta(\bar{G})-1$.

We will consider now the general case when $d \geq 1$ is fixed.

Theorem 4.2 For every fixed $d \geq 1$, Chromatic $d$-BlOCKen is polynomial-time solvable in complements of bipartite graphs.

Proof: Let $M$ be a maximum matching in $\bar{G}$. We distinguish two cases.

(1) $\mu(\bar{G}) \geq d+1$. By choosing $d+1$ edges of $M$ and adding the set $F$ of all non-edges between the saturated vertices, we obtain a clique of size $2 d+2$ and thus $\theta(\bar{G}+F) \leq \theta(\bar{G})-d$. Clearly the set $F$ of non-edges in $\bar{G}$ corresponds to a chromatic $d$-blocker $E^{\prime}$ in $G$ of size at most $\left(\begin{array}{c}2(d+1) \\ 2\end{array}\right)-(d+1)$. 
(2) $\mu(\bar{G})<d+1$. If there are at least $d+1$ non-saturated vertices, then we add all the non-edges between $d+1$ of them. This corresponds to a chromatic $d$-blocker of size $\left(\begin{array}{c}d+1 \\ 2\end{array}\right)$ in $G$. Otherwise, $G$ contains at most $d+2 \mu(\bar{G}) \leq 3 d$ vertices.

Hence, since $d$ is fixed, a minimum chromatic $d$-blocker can be found in polynomial time.

Let us now consider the case when $d \geq 1$ is not fixed. We will show that Chromatic $d$-BLOCKER is $N P$-hard in the complements of bipartite graphs by showing that the equivalent problem CLIQUE COVERING $d$-BLOCKER is $N P$-hard in bipartite graphs. In order to do so, we will use the following problem.

$P_{3}$-PARtition

Input: A bipartite graph $G=(V, E)$ on $3 n$ vertices such that $g(G) \geq 6$.

Question: Does there exist a partition $\mathcal{V}$ of $V$ into $n$ sets $\left\{V_{1}, \ldots, V_{n}\right\}$ such that for every $1 \leq i \leq$ $n,\left|V_{i}\right|=3$ and the graph $G\left[V_{i}\right]$ is isomorphic to $P_{3}$ ?

$P_{3}$-PARTition was shown to be $N P$-complete in $[15]$ without assuming that $g(G) \geq 6$. In fact, we can assume without loss of generality that $g(G) \geq 6$; this follows from the construction given in [15] and from the fact that for the three dimensional perfect matching problem (the problem that is used in the reduction in [15]), one can suppose that every element $e$ occurs in at least two triplets.

We start with the following lemma.

Lemma 4.3 $P_{3}$-PARTITION is NP-complete in bipartite graphs admitting a perfect matching.

Proof: Consider an instance $\mathcal{I}$ of $P_{3}$-PARTition consisting of a bipartite graph $G=(V, E)$ on $3 n$ vertices such that $g(G) \geq 6$. We build the following graph $G^{\prime}=\left(V^{\prime}, E^{\prime}\right)$ : with each vertex $v_{i} \in V$ we associate four vertices $v_{0}^{i}, v_{1}^{i}, v_{2}^{i}, v_{3}^{i}$ such that $G^{\prime}\left[\left\{v_{0}^{i}, v_{1}^{i}, v_{2}^{i}, v_{3}^{i}\right\}\right]$ is isomorphic to $P_{4}$; then we add an edge $v_{0}^{i} v_{0}^{j}$ in $G^{\prime}$ if and only if $v_{i} v_{j} \in E$. This transformation is clearly polynomial. Moreover $G^{\prime}$ is bipartite, $\left|V^{\prime}\right|=12 n, g\left(G^{\prime}\right) \geq 6$, and $M=\left\{v_{0}^{i} v_{1}^{i}, v_{2}^{i} v_{3}^{i} \mid v_{i} \in V\right\}$ is a perfect matching of $G^{\prime}$. Thus we obtain an instance $\mathcal{I}^{\prime}$ of $P_{3}$-PARTITION consisting of a bipartite graph $G^{\prime}=\left(V^{\prime}, E^{\prime}\right)$ with $\left|V^{\prime}\right|=3 p$ $(p=4 n)$ admitting a perfect matching.

Now suppose that $\mathcal{I}$ is a yes-instance, i.e., let $\left\{V_{1}, \ldots, V_{n}\right\}$ be a partition of $V$ such that $G\left[V_{i}\right]$ is isomorphic to $P_{3}$, for every $1 \leq i \leq n$. Then clearly $\left\{\left\{v_{0}^{a}, v_{0}^{b}, v_{0}^{c}\right\},\left\{v_{1}^{a}, v_{2}^{a}, v_{3}^{a}\right\},\left\{v_{1}^{b}, v_{2}^{b}, v_{3}^{b}\right\},\left\{v_{1}^{c}, v_{2}^{c}, v_{3}^{c}\right\} \mid V_{i}=\right.$ $\left.\left\{v_{a}, v_{b}, v_{c}\right\}, 1 \leq i \leq n\right\}$ is a partition of $V^{\prime}$ into $P_{3}$ 's, and hence $\mathcal{I}^{\prime}$ is a yes-instance.

Conversely, suppose that $\mathcal{I}^{\prime}$ is a yes-instance and let $\mathcal{V}^{\prime}$ be a partition; then necessarily each set $\left\{v_{1}^{a}, v_{2}^{a}, v_{3}^{a}\right\}, v_{a} \in V$, induces a $P_{3}$ of $\mathcal{V}^{\prime}$. It follows that the other $P_{3}$ 's of the partition are induced by the sets $\left\{v_{0}^{a}, v_{0}^{b}, v_{0}^{c}\right\}, v_{a}, v_{b}, v_{c} \in V$. Thus the corresponding sets $\left\{v_{a}, v_{b}, v_{c}\right\}$ induce each a $P_{3}$ in $G$ and hence $\mathcal{I}$ is a yes-instance.

Now we are ready to prove the following.

Theorem 4.4 Clique COVering $d$-Blocker is NP-hard in bipartite graphs.

Proof: Consider an instance $\mathcal{I}$ of $P_{3}$-PARTition consisting of a bipartite graph $G=(V, E)$ admitting a perfect matching such that $|V|=6 p, g(G) \geq 6$. We build an instance $\mathcal{I}^{\prime}$ of Clique COverING $d$ BLOCKER as follows: $G^{\prime}=\left(V^{\prime}, E^{\prime}\right)=G=(V, E), d=p$ and $k=2 p$. Since $G^{\prime}$ is bipartite and has a perfect matching, it follows that $\theta\left(G^{\prime}\right)=3 p$.

Now suppose that $\mathcal{I}$ is a yes-instance. Consider a partition $\left\{V_{1}, \ldots, V_{2 p}\right\}$ of $V$ such that $G\left[V_{i}\right]$ is isomorphic to $P_{3}$ for every $1 \leq i \leq 2 p$. Then consider the set of non-edges $\bar{B}=\left\{u w \mid V_{i}=\{u, v, w\}, 1 \leq\right.$ $i \leq 2 p\}$. Clearly $|\bar{B}|=2 p$ and $\theta\left(G^{\prime}+\bar{B}\right) \leq 2 p$; the clique covering in $G^{\prime}+\bar{B}$ consists of $2 p$ cliques 
$K^{i}$ of size 3 each induced by a set $\{u, v, w\}$, such that $V_{i}=\{u, v, w\}$, for $i=1, \ldots, 2 p$. Thus $\mathcal{I}^{\prime}$ is a yes-instance.

Conversely, assume now that $\mathcal{I}^{\prime}$ is a yes-instance. Let $\bar{B} \subseteq \bar{E}^{\prime},|\bar{B}| \leq 2 p$ such that $\theta\left(G^{\prime}+\bar{B}\right) \leq 2 p$. Since $G^{\prime}$ is bipartite and admits a perfect matching, $V^{\prime}$ can be partitioned into two stable sets $S_{1}^{\prime}, S_{2}^{\prime}$ such that $\left|S_{1}^{\prime}\right|=\left|S_{2}^{\prime}\right|=3 p$. In $G^{\prime}+\bar{B}$ each vertex set $S_{i}^{\prime}, i=1,2$, is necessarily covered by at most $2 p=\left|S_{i}^{\prime}\right|-p$ cliques, since $\theta\left(G^{\prime}+\bar{B}\right) \leq 2 p$. Thus $\bar{B}$ contains at least $p$ edges $u v$ such that $u, v \in S_{i}^{\prime}$ for $i=1,2$. Hence we have $|\bar{B}|=2 p$ and there are exactly $p$ edges $u v \in \bar{B}$ such that $u, v \in S_{i}^{\prime}$ for $i=1,2$. Notice that these edges must form a matching in $S_{i}^{\prime}$. Now since $g\left(G^{\prime}\right) \geq 6$, and hence $G^{\prime}$ does not contain any cycle $C_{4}$, the size of a maximum clique in $G^{\prime}+\bar{B}$ is three. Furthermore, since $\theta\left(G^{\prime}+\bar{B}\right) \leq 2 p$ and $\left|V^{\prime}\right|=6 p$, it follows that $G^{\prime}+\bar{B}$ contains exactly $2 p$ pairwise disjoint cliques, each of size three. Now to each $u v \in \bar{B}$ such that $u, v \in S_{i}^{\prime}$, for $i=1,2$, corresponds a path $P_{3}$ induced by $u, v, w$ in $G^{\prime}$. Since these $2 p$ paths are pairwise disjoint, they form a partition $\left\{V_{1}, \ldots, V_{2 p}\right\}$ of $V$ such that $G^{\prime}\left[V_{i}\right]$ is isomorphic to $P_{3}$ for $i=1, \ldots, 2 p$, and hence $\mathcal{I}$ is a yes-instance.

Corollary 4.5 Chromatic $d$-BLOCKER is NP-hard in the complements of bipartite graphs.

Notice that the complexity status of STABILITY $d$-BLOCKER in this case is still open.

\section{$5 \quad$ Bipartite graphs}

In this section, we will consider STABILITy $d$-BLOCKER in the case of general bipartite graphs and $d=1$. Let $G=(B, W, E)$ be a bipartite graph, where $B$ and $W$ denote the two sets of the bipartition. A vertex $v$ is called forced if every maximum stable set contains $v$. A vertex $v$ is called excluded if no maximum stable set contains $v$. A vertex which is neither forced nor excluded is called free. The set of all forced vertices in $G$ is denoted by $\mathcal{F}$ and the set of all excluded vertices will be denoted by $\mathcal{E}$. Clearly $\mathcal{F}, \mathcal{E}$ and $(B \cup W) \backslash(\mathcal{F} \cup \mathcal{E})$ form a partition of the vertex set $B \cup W$ and all the neighbors of a forced vertex are necessarily excluded vertices. This partition can be obtained in polynomial time for bipartite graphs. This follows from a result of [9] about König-Egerváry graphs, which include bipartite graphs.

We will need the following result which was shown in [10].

Theorem 5.1 [10] If $G=(B, W, E)$ is a bipartite graph, then the subgraph induced by $(B \cup W) \backslash(\mathcal{F} \cup \mathcal{E})$ (i.e., by its free vertices) contains a perfect matching.

As a consequence for a bipartite graph $G=(B, W, E)$ we have that $\alpha(G)=|\mathcal{F}|+\left|B^{\prime}\right|=|\mathcal{F}|+\left|W^{\prime}\right|$, where $B^{\prime}=B \cap((B \cup W) \backslash(\mathcal{F} \cup \mathcal{E}))$ and $W^{\prime}=W \cap((B \cup W) \backslash(\mathcal{F} \cup \mathcal{E}))$. This implies that when $\mathcal{F}=\emptyset$ we have $\mathcal{E}=\emptyset$, since otherwise either $\{v\} \cup B^{\prime}$ or $\{v\} \cup W^{\prime}$ is a stable set of size $\alpha(G)+1$, where $v$ is any vertex in $\mathcal{E}$.

We will also use the following result which was obtained in [7] for the more general case of weighted bipartite graphs, that we now state for unweighted bipartite graphs.

Theorem 5.2 [7] Let $G=(B, W, E)$ be a bipartite graph containing only free vertices. Then there exists a partition $\mathcal{V}=\left(V_{1}, \ldots, V_{q}\right)$ of $B \cup W$ such that a stable set $S \subseteq B \cup W$ is maximum if and only if for any $j \in\{1, \ldots, q\}$ either $S \cap V_{j}=B \cap V_{j}$ or $S \cap V_{j}=W \cap V_{j}$.

We will call a partition $\mathcal{V}$ as described in Theorem 5.2 a good partition. For such a partition the following properties were shown in [7]:

(i) $\mathcal{V}$ can be obtained in polynomial time; 
(ii) each graph $G\left[V_{i}\right]$ is connected and in addition we have $\left|W \cap V_{i}\right|=\left|B \cap V_{i}\right|$, for $i=1 \ldots, p$; notice that this implies that the cardinality of each set $V_{i}$ is even and at least two;

(iii) if there exists an edge between two vertices $u, v$ such that $u \in V_{i} \cap B$ and $v \in V_{j} \cap W, i \neq j$, then there exists no edge between vertices $x, y$ such that $x \in V_{i} \cap W$ and $y \in V_{j} \cap B$.

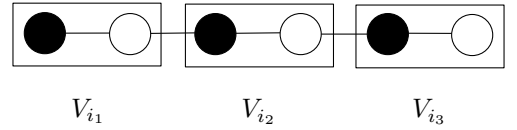

Figure 1: The graph $H_{1}$ with $i_{1}, i_{2}, i_{3} \in\{1, \ldots, q\}$.

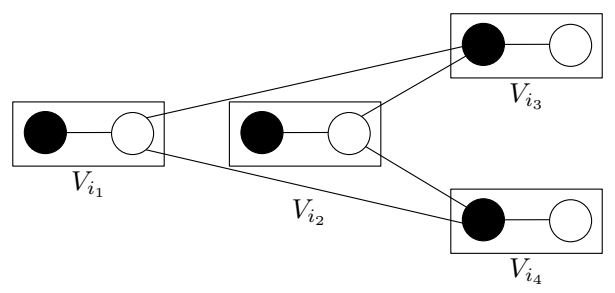

Figure 2: The graph $H_{2}$ with $i_{1}, \ldots, i_{4} \in\{1, \ldots, q\}$.

By using Theorem 5.2, we obtain the following.

Theorem 5.3 Let $G=(B, W, E)$ be a bipartite graph and let $\mathcal{V}=\left\{V_{1}, \ldots, V_{q}\right\}$ be a good partition of $V \backslash(\mathcal{F} \cup \mathcal{E})$. Then

(a) $s b_{1}(G)=1$ if and only if $|\mathcal{F}| \geq 2$;

(b) $s b_{1}(G)=2$ if and only if $|\mathcal{F}| \leq 1$ and

(b1) either $|\mathcal{F}|=1$;

(b2) or $\exists V_{i} \in \mathcal{V}$ such that $\left|V_{i}\right| \geq 4$;

(b3) or $G$ contains $H_{1}$ as a subgraph (see Figure 1);

(b4) or $\mathrm{G}$ contains $\mathrm{H}_{2}$ as a subgraph (see Figure 2);

(c) in all remaining cases:

(c1) $s b_{1}(G)=3$ if and only if there exist $V_{i}, V_{j} \in \mathcal{V}, i \neq j$, such that $x \in V_{i}, y \in V_{j}$ and $x y \in E$;

(c2) $s b_{1}(G)=4$ otherwise.

Proof: Consider a bipartite graph $G=(B, W, E)$. Recall that we may assume that $\alpha(G) \geq 3$.

(a) If $G$ contains at least two forced vertices, say $u, v$, then $\alpha(G+\{u v\})=\alpha(G)-1$. Conversely, if there exists a non-edge in $G$ which can be transformed into an edge, say $x y$, such that $\alpha(G+$ $\{x y\})=\alpha(G)-1$, then necessarily $x$ and $y$ are both forced vertices.

So we may assume now that $|\mathcal{F}| \leq 1$.

(b) Notice that, since $\alpha(G) \geq 3$ and $|\mathcal{F}| \leq 1$, we have $|B| \geq 2,|W| \geq 2$, and $|V \backslash(\mathcal{F} \cup \mathcal{E})| \geq 2$. From (a), we need to find at least two non-edges which must be changed into edges in order to decrease the stability number by at least one. Let $\mathcal{V}=\left(V_{1}, \ldots, V_{q}\right)$ be a good partition of $V \backslash(\mathcal{F} \cup \mathcal{E})$. We distinguish several cases: 
(b1) $\mathcal{F}=\{u\}$. Let $v_{1}, v_{2} \in V_{i}$, for some $i \in\{1, \ldots, q\}$, be such that $v_{1} \in B$ and $v_{2} \in W$. We claim that $\alpha\left(G+\left\{u v_{1}, u v_{2}\right\}\right)=\alpha(G)-1$. Indeed, it follows from the definition of forced vertices and Theorem 5.2 that any maximum stable set $S$ contains either $u, v_{1}$ or $u, v_{2}$.

So we may assume now that $\mathcal{F}=\emptyset$ (as mentioned previously we also have $\mathcal{E}=\emptyset$ ).

(b2) $\exists V_{i} \in \mathcal{V}$ such that $\left|V_{i}\right| \geq 4$. Let $v_{1}, \ldots, v_{4} \in V_{i}$ be such that $v_{1}, v_{2} \in B$ and $v_{3}, v_{4} \in W$. We claim that $\alpha\left(G+\left\{v_{1} v_{2}, v_{3} v_{4}\right\}\right)=\alpha(G)-1$. Indeed it follows from Theorem 5.2 that every maximum stable set $S$ in $G$ contains either $v_{1}, v_{2}$ or $v_{3}, v_{4}$.

It follows from property (ii) that we may assume from now on that $\left|V_{i}\right|=2$ for all $i \in\{1, \ldots, q\}$.

(b3) $G$ contains $H_{1}$ as a subgraph (see Figure 1). Let $\{x\}=V_{i_{1}} \cap B,\{u\}=V_{i_{1}} \cap W,\{y\}=V_{i_{2}} \cap B$, $\{z\}=V_{i_{2}} \cap W$ and $\{t\}=V_{i_{3}} \cap W$. We claim that $\alpha(G+\{x y, z t\})=\alpha(G)-1$. Suppose the claim does not hold and let $S$ be a maximum stable set in $G+\{x y, z t\}$ such that $|S|=\alpha(G)$. Since $S$ is also a maximum stable set in $G$, it follows that $S$ has the properties mentioned in Theorem 5.2. Since $x$ and $u$ are both adjacent to $y$, it follows that $z$ must belong to $S$. This necessarily implies that $S \cap V_{i_{3}}=\emptyset$, a contradiction. Thus, such a stable set $S$ does not exist.

So we may assume now that $G$ does not contain $H_{1}$ as a subgraph.

(b4) $G$ contains $H_{2}$ as a subgraph (see Figure 2). Let $\{x\}=V_{i_{1}} \cap B,\{v\}=V_{i_{1}} \cap W,\{y\}=V_{i_{2}} \cap B$, $\{u\}=V_{i_{2}} \cap W,\{z\}=V_{i_{3}} \cap W$ and $\{t\}=V_{i_{4}} \cap W$. We claim that $\alpha(G+\{x y, z t\})=\alpha(G)-1$. Suppose the claim does not hold and, as in (3), let $S$ be a maximum stable set in $G+\{x y, z t\}$ such that $|S|=\alpha(G)$. First assume that $S$ contains $x$. Then it must contain $u$. This implies that $S$ must contain $z$ and $t$, a contradiction. So we may assume now that $S$ does not contain $x$ and hence $S$ must contain $v$. But this implies again that $S$ must contain $z$ and $t$, a contradiction. Thus such a stable set $S$ does not exist.

So we may assume now that $G$ does not contain $H_{2}$ as a subgraph.

(c) We show that in all remaining cases we need to find at least three non-edges which must be changed into edges in order to decrease the stability number by at least one. Suppose by contradiction that two non-edges are sufficient. Since, from Theorem 5.2, $B$ and $W$ are two disjoint maximum stable sets, it follows that one of these non-edges has its endvertices in $B$, say $x_{B}, y_{B}$, and the other non-edge has its endvertices in $W$, say $x_{W}, y_{W}$.

Here we need to use the following notation: for each $V_{i}=\{x, y\} \in \mathcal{V}$, the vertex $y$ will be denoted by $m(x)$, i.e. $V_{i}=\{x, m(x)\}$.

Let $i_{1} \neq i_{2}$ be such that $x_{B} \in V_{i_{1}}$ and $y_{B} \in V_{i_{2}}$. First assume that $x_{B}$ is adjacent to $m\left(y_{B}\right)$. Since $G$ does not contain $H_{1}$ as a subgraph and since there must be a non-edge between $m\left(x_{B}\right)$ and $y_{B}$ (see property (iii)), it follows that $N_{G}\left(m\left(x_{B}\right)\right) \cap B=\left\{x_{B}\right\}$. But now in $G+\left\{x_{B} y_{B}, x_{W} y_{W}\right\}$ the stable set $S$ such that $S \cap V_{i_{1}}=\left\{m\left(x_{B}\right)\right\}$ and $S \cap V_{j}=B \cap V_{j}$ for $j=1, \ldots, q, j \neq i_{1}$, clearly has size $\alpha(G)$, a contradiction. Therefore $x_{B} m\left(y_{B}\right) \notin E$. Similarly, we can show that $y_{B} m\left(x_{B}\right) \notin E, x_{W} m\left(y_{W}\right) \notin E$ and $y_{W} m\left(x_{W}\right) \notin E$.

Let $V_{i_{3}}, \ldots, V_{i_{r}} \in \mathcal{V}$ be such that $m\left(y_{B}\right) u_{i_{j}} \in E$ for $j=3, \ldots, r$, where $\left\{u_{i_{j}}\right\}=V_{i_{j}} \cap B$. Since $G$ does not contain $H_{1}$ and since there must be non-edges between $y_{B}$ and all the vertices $m\left(u_{i_{j}}\right)$ (see property (iii)), it follows that $N_{G}\left(m\left(u_{i_{j}}\right)\right) \cap B=\left\{u_{i_{j}}\right\}$, for $j=3, \ldots, r$. Now consider the set $S$ defined as follows: for $j=2, \ldots, r, S \cap V_{i_{j}}=W \cap V_{i_{j}}$ and for all remaining sets $V_{i} \in \mathcal{V}$ we take $S \cap V_{i}=B \cap V_{i}$. Since $S$ is not a stable set in $G+\left\{x_{B} y_{B}, x_{W} y_{W}\right\}$ (otherwise we would have $|S|=\alpha(G)$ ), it follows that $x_{W}, y_{W} \in V_{i_{2}} \cup \ldots \cup V_{i_{r}}$. Assume $x_{W} \in V_{i_{2}}$ (the case where $y_{W} \in V_{i_{2}}$ is symmetric), and consider the set $S$ defined as follows: $S \cap V_{i_{2}}=\left\{y_{B}\right\}$, and for all remaining sets $V_{i} \in \mathcal{V}$ we take $S \cap V_{i}=W \cap V_{i} . S$ is a stable set of size $\alpha(G)$ in $G+\left\{x_{B} y_{B}, x_{W} y_{W}\right\}$, since $N_{G}\left(y_{B}\right) \cap W=\left\{m\left(y_{B}\right)\right\}$ (otherwise $G$ would contain $H_{1}$ as a subgraph). Therefore, without loss of generality, we may assume that $x_{W} \in V_{i_{3}}$ and $y_{W} \in V_{i_{4}}$. 
Now, let $V_{j_{1}}, \ldots, V_{j_{s}} \in \mathcal{V}$ be such that $m\left(x_{B}\right) u_{j_{i}} \in E$ for $i=1, \ldots, s$, where $\left\{u_{j_{i}}\right\}=V_{j_{i}} \cap B$. We proceed exactly as before: since $G$ does not contain $H_{1}$ and since there must be non-edges between $x_{B}$ and all the vertices $m\left(u_{j_{i}}\right)$ (see property (iii)), it follows that $N_{G}\left(m\left(u_{j_{i}}\right)\right) \cap B=\left\{u_{j_{i}}\right\}$, for $i=1, \ldots, s$. Let $S^{\prime}$ be the set obtained by choosing for $i=1, \ldots, s, S^{\prime} \cap V_{j_{i}}=W \cap V_{j_{i}}$, $S^{\prime} \cap V_{i_{1}}=\left\{m\left(x_{B}\right)\right\}$ and for the remaining sets $V_{i} \in \mathcal{V}, S^{\prime} \cap V_{i}=B \cap V_{i}$. Since $S^{\prime}$ is not a stable set in $G+\left\{x_{B} y_{B}, x_{W} y_{W}\right\}$ (otherwise we would have $\left|S^{\prime}\right|=\alpha(G)$ ) it follows that $x_{W}, y_{W} \in$ $V_{j_{1}} \cup \ldots \cup V_{j_{s}}$. Thus $V_{i_{3}}, V_{i_{4}} \in\left\{V_{j_{1}}, \ldots, V_{j_{s}}\right\}$. But now we obtain $H_{2}$ by taking $V_{i_{1}}, V_{i_{2}}, V_{i_{3}}, V_{i_{4}}$, a contradiction. Hence two non-edges are not sufficient to decrease the stability number by at least one.

Now let us distinguish two cases:

(c1) There exist two distinct sets $V_{i}, V_{j} \in \mathcal{V}$ such that $\{x\}=V_{i} \cap B,\{y\} \in V_{j} \cap W$ and $x y \in E$. Clearly, any maximum stable set $S$ in $G+\{y m(x), m(x) m(y), x m(y)\}$ satisfies $|S|=\alpha(G)-1$.

(c2) There are no edges between any two distinct sets $V_{i}, V_{j} \in \mathcal{V}$. Clearly changing the four nonedges between two distinct sets $V_{i_{1}}, V_{i_{2}}$ into edges is sufficient to decrease the stability number by one. We will show now that changing four non-edges into edges is also necessary. Since, from Theorem 5.2, $B$ and $W$ are two disjoint maximum stable sets in $G$, it follows that we need to change one non-edge between two vertices in $B$, say $x, y$, and one non-edge between two vertices in $W$, say $z, t$, into edges. Assume w.l.o.g. that $t \neq m(y)$. Now consider in $G+\{x y, z t\}$ the following two disjoint stable sets of size $\alpha(G): S_{1}=(B \backslash\{x, m(t)\}) \cup\{m(x), t\}$ and $S_{2}=(W \backslash\{m(x), t\}) \cup\{x, m(t)\}$. Thus we need to change at least two more non-edges into edges.

Theorem 5.3 immediately implies the following result.

Corollary 5.4 STABILITY 1-BLOCKER is polynomial-time solvable for bipartite graphs.

\section{Conclusion}

In this paper we considered two problems, namely Chromatic $d$-BLOCKER and STABILITy $d$-BLOCKER, and investigated their computational complexity in different classes of graphs. The results that we obtained are summarized in Table 1 below.

\begin{tabular}{|l|c|c|c|c|c|}
\hline Graph class & General & Split & Threshold & $\begin{array}{c}\text { Complement } \\
\text { of bipartite }\end{array}$ & Bipartite \\
\hline Chromatic $d$-BLOCKER & $\begin{array}{c}N P \text {-hard } \\
\forall d \geq 1 \text { fixed }\end{array}$ & $P, \forall d \geq 1$ fixed & $P$ & $\begin{array}{c}N P \text {-hard } \\
P, \forall d \geq 1 \text { fixed }\end{array}$ & $P$ (trivial) \\
\hline StABILITy $d$-BLOCKER & $\begin{array}{c}N P \text {-hard } \\
\forall d \geq 1 \text { fixed }\end{array}$ & $P, \forall d \geq 1$ fixed & $?$ & $P$ (trivial) & $P, d=1$ \\
\hline
\end{tabular}

Table 1: Results concerning Chromatic $d$-Blocker and Stability $d$-Blocker.

There are still many open questions left with respect to these two problems. Let us state here those that we consider as the most interesting ones.

(a) What is the complexity of StABILITy $d$-BLOCKER in threshold graphs if $d$ is not fixed? 
(b) What is the complexity of Chromatic $d$-Blocker and Stability $d$-Blocker in split graphs when $d$ is not fixed?

(c) What is the complexity of StABILITy $d$-BLOCKER in bipartite graphs when $d \geq 2$ is fixed respectively when $d$ is not fixed?

\section{Acknowledgements}

This work has been supported by the Groupe de Recherche - Recherche Opérationnelle (GDR-RO) of CNRS, which is gratefully acknowledged.

The authors would also like to express their gratitude to the anonymous referees for their constructive comments and remarks, which helped to improve the readability of this paper.

\section{References}

[1] A. Asratian, T.M.J. Denley, R. HäGgkvist, Bipartite Graphs and Their Applications, Cambridge University Press (1998).

[2] A. Bar-Noy, S. Khuller, B. Schieber, The complexity of finding most vital arcs and nodes, Technical Report CS-TR-3539, University of Maryland (1995).

[3] C. Bazgan, S. Toubaline, Z. Tuza, The most vital nodes with respect to independent set and vertex cover, Discrete Applied Mathematics 159(17) (2011) 1933-1946.

[4] C. Bazgan, S. Toubaline, Z. Tuza, Complexity of the most vital nodes for Independent Set on tree structures Proceedings of the $21^{\text {st }}$ International Workshop on Combinatorial Algorithms (IWOCA 2010), LNCS 6460 (2010) 154-166.

[5] C. Bazgan, S. Toubaline, D. Vanderpooten, Complexity of determining the most vital elements for the p-median and p-center location problems, Journal of Combinatorial Optimization 25(2) (2013) 191-207.

[6] C. Bazgan, S. Toubaline, D. Vanderpooten, Critical edges/nodes for the minimum spanning tree problem: complexity and approximation Journal of Combinatorial Optimization 26 (2013) 178-189.

[7] C. Bentz, M.-C. Costa, C. Picouleau, B. Ries, D. de Werra, d-Transversals of Stable Sets and Vertex Covers in Weighted Bipartite Graphs, Journal of Discrete Algorithms 17 (2012) 95-102.

[8] R.L. Brooks, On colouring the nodes of a network, Proc. Cambridge Philosophical Society, Math. Phys. Sci. 37 (1941) 194-197.

[9] M. Chlebík, J. Chlebíková, Crown reductions for the Minimum Weighted Vertex Cover problem, Discrete Applied Mathematics 156(3) (2008) 292-312.

[10] M.-C. Costa, D. de Werra, C. Picouleau, Minimum $d$-blockers and $d$-transversals in graphs, Journal of Combinatorial Optimization 22(4) (2011) 857-872.

[11] J. F. Fink, M.S. Jacobson, L. F. Kinch, J. Roberts, The bondage number of a graph, Discrete Mathematics 86(1-3) (1990) 47-57. 
[12] G. N. Frederickson, R. Solis-ObA, Increasing the weight of minimum spanning trees, Proceedings of the $7^{\text {th }}$ ACM-SIAM Symposium on Discrete Algorithms (SODA 1996) 539-546.

[13] M.R. Garey, D.S. Johnson, Computers and tractability: a guide to the theory of NPcompleteness, Freeman, New York (1979).

[14] L. Khachiyan, E. Boros, K. Borys, K. Elbassioni, V. Gurvich, G. Rudolf, J. Zhao, On short paths interdiction problems : total and node-wise limited interdiction, Theory of Computing Systems 43(2) (2008) 204-233.

[15] J. Monnot, S. Toulouse, The path partition problem and related problems in bipartite graphs, Operations Research Letters 35(5) (2007) 677-684.

[16] B. Ries, C. Bentz, C. Picouleau, D. de Werra, M.-C. Costa, R. Zenklusen, Blockers and Transversals in some subclasses of bipartite graphs : when caterpillars are dancing on a grid, Discrete Mathematics 310(1) (2010) 132-146.

[17] S. Toubaline, Détermination des éléments les plus vitaux pour des problèmes de graphes, $P h D$ Thesis, Université Paris-Dauphine (2010).

[18] P. TuRÁn, On an extremal problem in graph theory (in Hungarian), Matematikai s Fizikai Lapok 48 (1941) 436-452.

[19] D.B. West, Introduction to Graph Theory, Second Edition, Prentice Hall (2001).

[20] R. K. Wood, Deterministic network interdiction, Mathematical and Computer Modeling 17(2) (1993) 1-18.

[21] R. Zenklusen, Matching Interdiction, Discrete Applied Mathematics 158 (2010) 1676-1690.

[22] R.Zenklusen, B. Ries, C. Picouleau, D. de Werra, M.-C. Costa, C. Bentz, Blockers and Transversals, Discrete Mathematics 309(13) (2009) 4306-4314. 